\title{
Heart Rate Variability Predicts Emotional Flexibility in Response to Positive Stimuli
}

\author{
Tomomi Fujimura ${ }^{1,2}$, Kazuo Okanoya ${ }^{1,2,3 *}$ \\ ${ }^{1}$ Japan Science Technology Agency, ERATO, Okanoya Emotional Information Project, Saitama, Japan \\ ${ }^{2}$ RIKEN Brain Science Institute, Saitama, Japan \\ ${ }^{3}$ Department of Life Sciences, Graduate School of Arts and Sciences, The University of Tokyo, Tokyo, Japan \\ Email: "kazuookanoya@gmail.com \\ Received May $7^{\text {th }}$, 2012; revised June $7^{\text {th }}$, 2012; accepted July $10^{\text {th }}$, 2012
}

\begin{abstract}
Flexible adaptation to constantly changing environments is linked to mental health and psychological functioning. Heart rate variability (HRV), an index of autonomic flexibility, has been implicated in emotional flexibility, the ability to generate contextually dependent emotional responses in accordance with situational demands. The current study investigated whether HRV during rest is associated with experienced emotion, one of the measures of emotional flexibility. To assess experienced emotion in response to changing events, three types of stimuli sets were created by presenting two stimuli successively. First, two stimuli represented the same valence (i.e., negative/negative or positive/positive). Second, two stimuli represented opposite valences (i.e., negative/positive or positive/negative). Third, a neutral stimulus was followed by negative or positive stimulus (i.e., neutral/negative or neutral/positive). Psychological ratings for experienced emotion to the second stimulus were collected with regard to valence and arousal. The results showed that subjects with lower resting HRV experienced more aroused states in response to successive positive stimuli. Resting HRV may be a proxy of emotional flexibility indexed by subjective arousal states to positive events.
\end{abstract}

Keywords: Heart Rate Variability; Emotional Flexibility; Psychological Ratings

\section{Introduction}

Life involves constantly changing events in the external environment. Rapid and adequate adaptation to these changes is vital for organisms to survive. Recent research has focused on emotional flexibility, the ability to flexibly respond to positive and negative events and to regulate emotion effectively (Waugh, Thompson, \& Gotlib, 2011; Westphal, Seivert, \& Bonanno, 2010). Individuals with greater emotional flexibility have positive reactivity to positive events and negative reactivity to negative events, in accordance with situational demands. These context-dependent emotional responses are characterized by flexible autonomic, somatic, and psychological responses to changing events.

Heart rate variability (HRV), an index of autonomic flexibility, is positively associated with good psychological and physiological functioning (Thayer \& Lane, 2000). HRV is calculated by R-R wave intervals produced by electrocardiographic data. In healthy individuals, heart rate fluctuates during resting states due to the different frequency characteristics of sympathetic and parasympathetic neural modulations of heart rate. Therefore, HRV is indicative of autonomic balance between sympathetic and parasympathetic activity. Low HRV is associated with a number of psychological disorders characterized by poor emotion regulation and behavioral inflexibility (see Thayer \& Brosschot, 2005 for a review). Given that emotional flexibility can be defined by autonomic and behavioral reactivity, it is possible that HRV assesses an individual's emotional flexibility, or how well an individual responds to emo-

"Corresponding author. tional events and generates contextually dependent emotional responses.

Individual differences in regulated emotional response can be predicted by baseline HRV (see Appelhans \& Luecken, 2006 for a review). For example, subjects with low resting HRV showed a more exaggerated startle reflex potentiated by the threat of shock compared with subjects with high resting HRV (Melzig, Weike, Hamm, \& Thayer, 2009). Furthermore, the emotion-modulated startle reflex was higher in subjects with low HRV than in those with high HRV and subjects with low HRV showed no difference in startle magnitude during negative, positive, and neutral foregrounds (Ruiz-Padial, Sollers, Vila, \& Thayer, 2003). This evidence suggests that individuals with low resting HRV have a tendency to produce an exaggerated startle reflex regardless of valence, reflecting an inappropriate response to situational demands.

Attention allocation, which involves the selection of meaningful information from the external environment, is also associated with resting HRV (Thayer \& Brosschot, 2005). A previous study reported that individuals with low HRV could not shift their attention away from negative stimuli, resulting in prolonged response initiation after the presentation of negative stimuli (Krypotos, Jahfari, van Ast, Kindt, \& Forstmann, 2011). In relation to this, Johnsen, Thayer, Laberg, Wormnes, Raadal, Skaret, Kvale, and Berg (2003) found increased attentional bias to threat-related words (i.e., dental-related words for dental phobics) in subjects with low HRV. This evidence indicates that HRV during rest is associated with sensitivity to threat signals. Because attentional control is important for adapting to a constantly changing environment, excessive sensitivity to 
threat signals interferes with the processing of subsequent events, leading to emotional inflexibility.

The aforementioned studies have revealed that resting HRV predicts individual differences in emotional flexibility by measuring reflexes and attention to emotional stimuli directly or indirectly. However, it remains unknown whether subjective emotions measured by psychological ratings are related to resting HRV, although subjective emotion is one of the indices to assess emotional flexibility. Waugh et al. (2011) demonstrated that high-resilient people who exhibited emotional flexibility, compared to low-resilient people, showed a greater difference in self-reported affect between current negative and positive pictures. Given that emotional flexibility is characterized by context-dependent experienced emotion, HRV, an index of flexibility, is associated with experienced emotion as measured by psychological ratings. That is, people with low HRV may show poor emotional experiences to positive and negative stimuli.

The present study aimed to examine whether resting HRV predicts individual differences in the experience of subjective emotion. To assess emotional flexibility, experienced emotion was evaluated in response to target stimuli followed by three types of prime stimuli. In the first case, the prime and target stimuli represented the same valence (i.e., negative/negative or positive/positive). In the second case, the prime and target stimuli represented opposite valences (i.e., negative/positive or positive/negative). In the third case, a neutral prime stimulus was followed by negative or positive target stimuli (i.e., neutral/negative or neutral/positive). Participants were asked to rate their experienced emotion when viewing the picture that was presented second (i.e., the target picture). We hypothesized that subjects with high resting HRV, in comparison with subjects with low resting HRV, would have stronger experiences of pleasantness or unpleasantness in response to the target stimulus, regardless of which stimulus was presented first. In addition to valence ratings, arousal ratings for experienced emotion were collected. Individuals with low resting HRV are characterized by chronic autonomic hyperarousal and diminished response habituation (Friedman \& Thayer, 1998). Accordingly, experienced arousal may be exaggerated in individuals with low resting HRV. Furthermore, this tendency may be prominent in the congruent condition in which same-valence stimuli were presented successively because individuals with low HRV are unlikely to habituate these emotional stimuli. In the present study, we expected that individual difference in resting HRV would translate into positive and negative reactivity, assessed by experienced emotion.

\section{Method}

\section{Participants}

Forty-three adults (24 men and 19 women; mean $\pm S D$ age, $25.02 \pm 6.74$ years) participated in the study. The participants were recruited by advertisements placed with an intermediary company, and their occupational backgrounds varied widely. They received compensation for participating in the experiment. All of the participants had normal or corrected-to-normal vision.

\section{Stimulus Material}

The stimuli were 35 pictures selected from the International
Affective Picture System (IAPS: Lang, Bradley, \& Cuthbert, 2008), consisting of 10 pleasant, 5 neutral, and 10 unpleasant pictures. The mean of valence/arousal ratings were: 7.63/5.10 for the pleasant pictures, 4.99/2.45 for neutral pictures, and 2.73/5.23 for unpleasant pictures. Arousal ratings were equivalent for pleasant and unpleasant pictures. All pictures were presented in full color.

\section{Apparatus and Physiological Measurement}

Experimental events were controlled by a program written in Inquisit 3.0 (Millisecond) and were implemented on a computer (Vostro 420, Dell) using the Microsoft Windows XP operating system. Stimuli were presented on a 19-inch LCD monitor (E1902S, Iiyama; $1024 \times 768$ pixels, $75 \mathrm{~Hz}$ refresh rate) and subtended a visual angle of about $20.8^{\circ} \times 28.1^{\circ}$.

The electrocardiogram (ECG) was recorded with a data acquisition system (MP150 system; BIOPAC systems Inc., Goleta, CA) with electrodes placed in a Lead II configuration. The ECG signals were .5 to $35 \mathrm{~Hz}$ bandpass filtered and amplified using a BIOPAC amplifier (ECG100C; BIOPAC systems Inc., Goleta, CA) and were digitized with a sampling rate of 1000 $\mathrm{Hz}$.

\section{Procedure}

Experiments were conducted individually in an electronically shielded and sound-attenuated room. Upon arrival, participants were told that the electrodes were harmless and that they could withdraw from the experiment at any time. All participants completed the informed consent form and successfully participated in the experiment. After all sensors were attached, task instructions were provided.

To assess baseline values of HRV in a resting period, ECG was measured for 5 min during a resting state. Participants were asked to relax into a chair but not to close their eyes to avoid falling into sleep.

After the measurement of resting HRV, a picture evaluation task was conducted. For each trial, two pictures were consecutively displayed. To assess experienced emotion elicited by the second picture (i.e., the target stimulus) preceded by the first picture (i.e., the prime stimulus), three types of conditions including pairings of pictures were produced. The first pairing consisted of negative/negative or positive/positive stimuli. The second pairing consisted of negative/positive or positive/negative stimuli. The third pairing consisted of neutral/negative or neutral/positive stimuli. These six pairings were arranged into three blocks according to the hedonic content of the prime stimuli (positive, neutral, and negative blocks). Each prime stimulus was presented two times within a block. In each block, 10 pairings of pictures were presented, including five positive pictures and five negative pictures as target stimuli. The target stimuli were identical across the three blocks. The three blocks were randomly conducted across participants.

A trial began with a 2-s fixation point followed by two successive pictures presented for $6 \mathrm{~s}$ each. After a 6-s blank screen, the rating screen was displayed until the participant responded. The inter-trial interval was $6 \mathrm{~s}$. The psychological rating task was the $9 \times 9$ Affect Grid, which assessed affect along the dimensions of valence and arousal (Russell, Weiss, \& Mendelsohn, 1989). Participants were asked to rate how they felt when 
T. FUJIMURA, K. OKANOYA

(a)

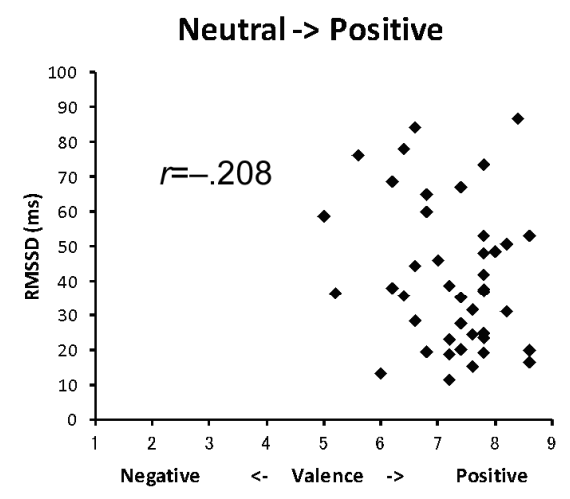

Neutral $->$ Negative

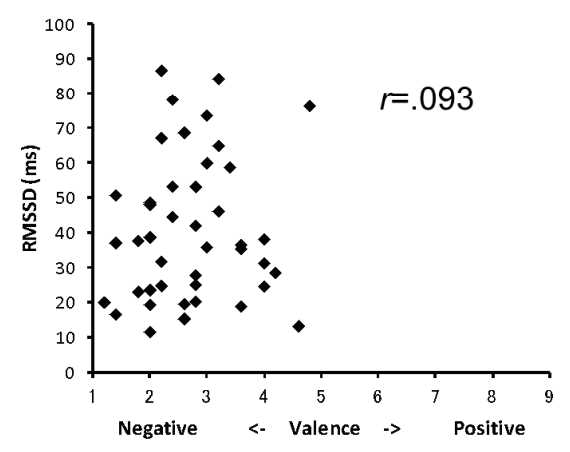

(b)

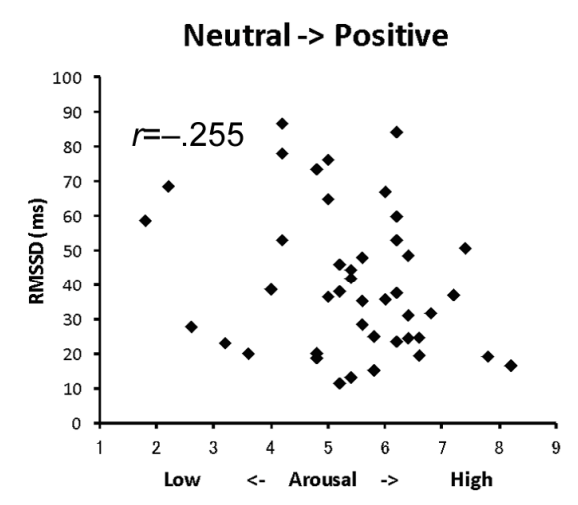

Neutral -> Negative

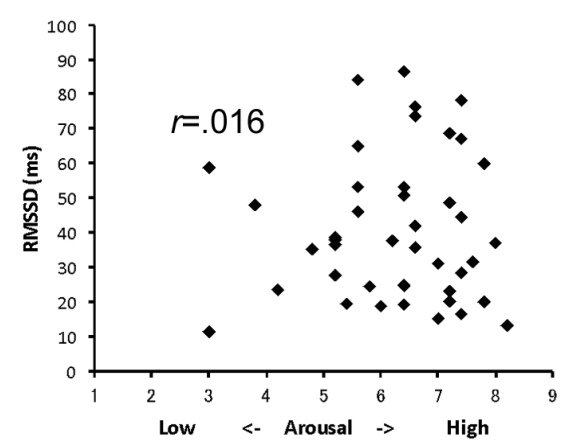

Valence Ratings

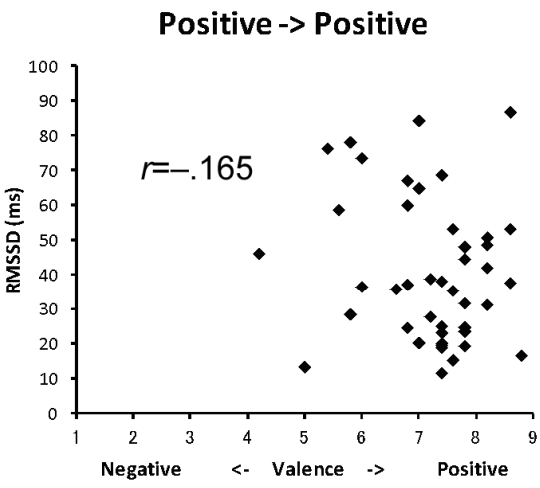

Negative $>>$ Negative

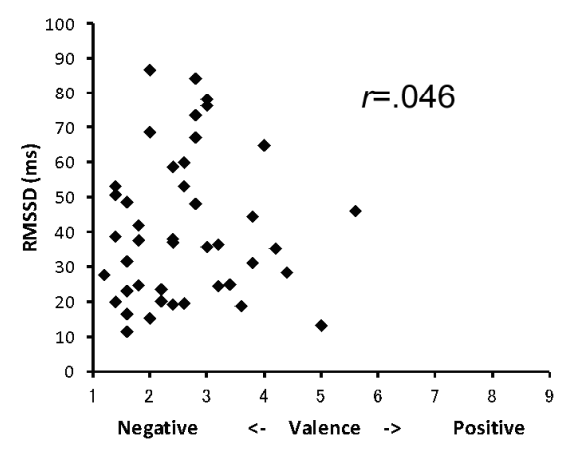

Arousal Ratings

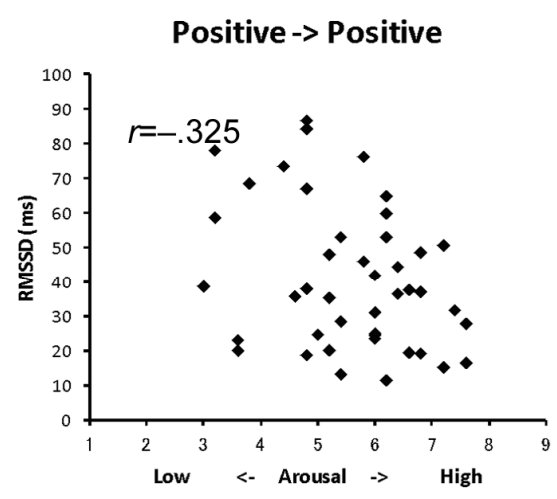

Negative -> Negative

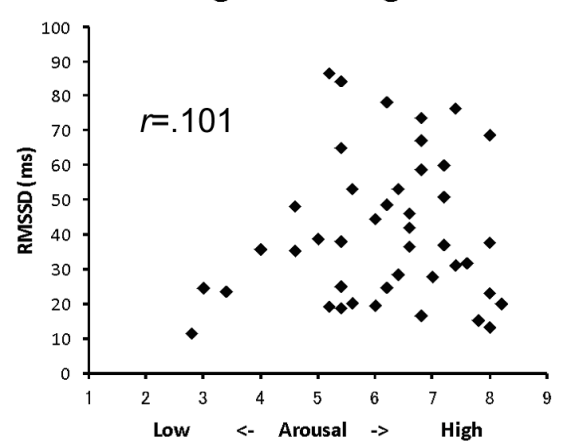

Negative -> Positive

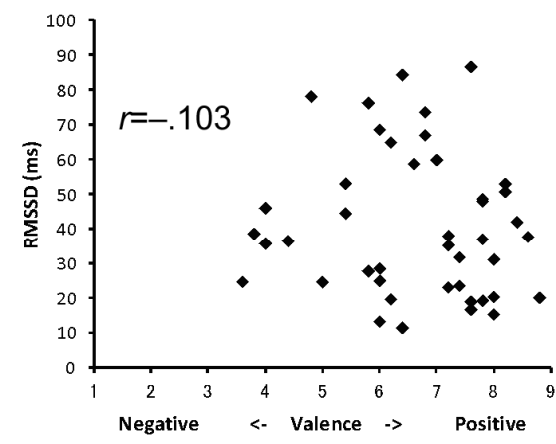

Positive -> Negative

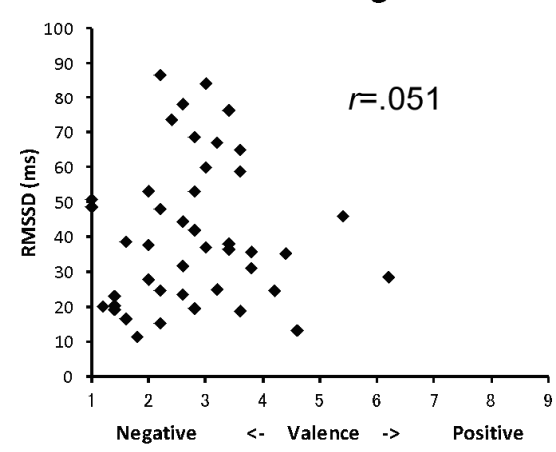

\section{Negative -> Positive}

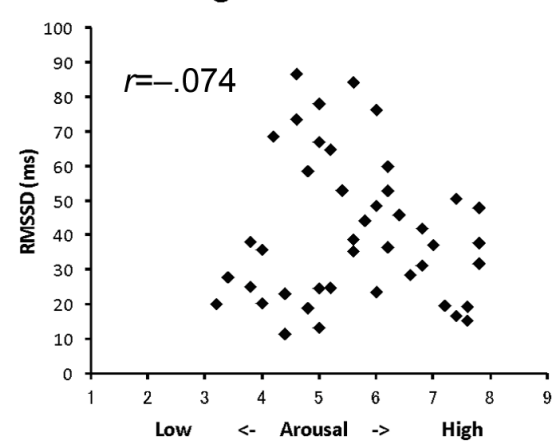

Positive -> Negative

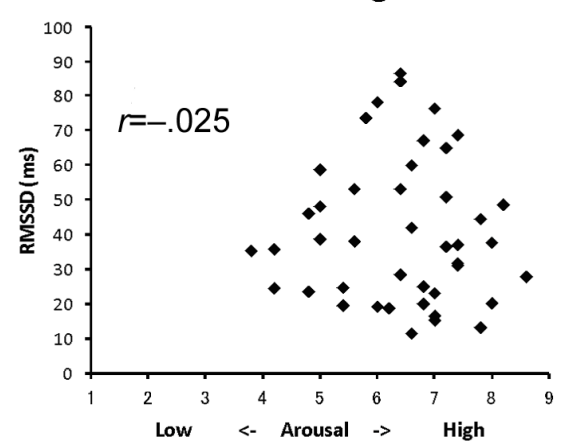

Figure 1.

Scatter plots for functions of psychological ratings and RMSSD and correlation coefficients for each relation: (a) Valence ratings and RMSSD; (b) Arousal ratings and RMSSD. 
they viewed the picture that was presented second by indicating a square on a two-dimensional emotional space using a computer mouse. All the participants used the right hand to rate the feeling.

\section{Data analysis}

To evaluate HRV, inter-beat intervals (IBI) were derived from ECG signals using software for analysis (Acknowledge 4.1; BIOPAC systems Inc., Goleta, CA). First, IBI were checked in a tachograph and corrected if the R-wave triggers were misplaced. To evaluate a time domain of HRV, the root mean square successive difference (RMSSD) (see the Task Force of the European Society of Cardiology and the North American Society of Pacing Electrophysiology, 1996) was calculated from IBI for a 5-min resting period for each participant.

For the psychological ratings, valence and arousal ratings on a 9-point scale were collected for each trial.

\section{Results}

The mean of the RMSSD calculated by IBI during the 5-min resting period across participants was $40.84 \mathrm{~ms}(S D=20.53)$. Figure 1 shows scatter plots by function of valence or arousal ratings and RMSSD. To determine the relationship between subjective states elicited by emotional stimuli and baseline HRV, correlation coefficients were calculated for each condition. We found a significant negative correlation between arousal ratings for positive stimuli preceded by positive stimuli and RMSSD $(r=-.325 ; t(41)=2.20, p<.05)$. There were no other significant correlations.

\section{Discussion}

In the current study, we investigated whether resting HRV is associated with experienced emotion elicited by multiple emotional events. The results showed that individuals with lower HRV experienced more aroused states when viewing successive positive stimuli. This is the first evidence indicating an association between resting HRV and subjective experienced emotion by measuring psychological ratings.

Resting HRV was negatively associated only with arousal ratings, not valence ratings, when viewing successive two positive pictures. This result showed that lower resting HRV led to higher arousal states in response to multiple positive stimuli. This finding is consistent with previous findings suggesting that individuals with low HRV are in states of hyperarousal and diminished habituation by various autonomic measures (Friedman \& Thayer, 1998). Furthermore, low HRV during rest has been shown to resist habituation, even to non-threat stimuli, due to hypervigilance (Thayer, Friedman, Borkovec, Johnsen, \& Molina, 2000). According to this evidence, subjects with low HRV fail to adapt to successive positive stimuli, resulting in feelings of relatively high arousal instead of pleasantness. This finding suggests that individuals with low HRV show poor habituation and assess their subjective emotional states as aroused. Resting HRV may be associated with the ability to control subjective arousal states rather than to enhance pleasantness or unpleasantness when confronting emotional stimuli. Another explanation of the relationship between reduced HRV and high arousal states is that HRV is positively related to good emotion regulation (Porges, 2007; Porges \& Byrne, 1992;
Thayer \& Lane, 2000, 2009). Emotion regulation involves the process by which people manage both negative and positive emotions (Gross, 1998). Successful emotion regulation, by either reappraisal or suppression, has been shown to lead to increased vagally mediated HRV (Butler, Wilhelm, \& Gross, 2006; Di Simplicio, Costoloni, Western, Hanson, Taggart, \& Harmer, 2011). Furthermore, participants with a high baseline of vagally mediated HRV spontaneously use emotion regulation strategies more often during emotional conversation than participants with a low baseline of vagally mediated HRV (Butler et al., 2006). Consistent with these findings, our results suggest that high arousal states in response to positive stimuli result from failures of emotion regulation in subjects with lower HRV.

Notably, our findings indicated that resting HRV was related to emotional experience elicited by positive stimuli, not negative stimuli, although most previous studies have shown an association between resting HRV and the processing of threatening or negative stimuli (Appelhans \& Luecken, 2006; Johnsen et al., 2003; Krypotos et al., 2011; Melzig et al., 2009). However, individuals with low resting HRV produced an exaggerated emotion-modulated startle reflex in response to neutral and positive pictures (Ruiz-Padial et al., 2003). This tendency could be due to sensitivity to non-threat signals. In this study, it is possible that the misperception of safety (i.e., positive stimuli) in individuals with low HRV caused higher arousal states compared to individuals with high HRV. This finding provides evidence of emotional inflexibility in response to multiple positive stimuli. Thus, resting HRV may predict emotional flexibility indexed by the experience of high arousal to positive events.

In summary, the current study revealed that resting HRV is a good proxy of emotional flexibility indexed by subjective experienced emotion. Individuals with lower HRV experienced more arousal states when faced with multiple positive stimuli. This may be due to emotional inflexibility derived from sensitivity to non-threat signals and/or failure of emotion regulation. HRV may indicate how individuals respond to positive events effectively and successfully. Future research should investigate the use of resting HRV as a proxy of emotional flexibility by measuring psychological, autonomic, and behavioral indices. Resting HRV provides useful information to understand individual differences in emotional flexibility.

\section{Acknowledgements}

We thank Kentaro Katahira and for assistance with editing this manuscript.

\section{REFERENCES}

Appelhans, B., \& Luecken, L. (2006). Heart rate variability as an index of regulated emotional responding. Review of General Psychology, 10, 229-240. doi:10.1037/1089-2680.10.3.229

Butler, E. A., Wilhelm, F. H., \& Gross, J. J. (2006). Respiratory sinus arrhythmia, emotion, and emotion regulation during social interaction. Psychophysiology, 43, 612-622. doi:10.1111/j.1469-8986.2006.00467.x

Di Simplicio, M., Costoloni, G., Western, D., Hanson, B., Taggart, P., \& Harmer, C. J. (2011). Decreased heart rate variability during emotion regulation in subjects at risk for psychopathology. Psychological Medicine, 1-9.

Friedman, B. H., \& Thayer, J. F. (1998). Autonomic balance revisited: 
Panic anxiety and heart rate variability. Journal of Psychosomatic Research, 44, 133-151. doi:10.1016/S0022-3999(97)00202-X

Gross, J. (1998). The emerging field of emotion regulation: An integrative review. Review of General Psychology, 2, 271-299. doi:10.1037/1089-2680.2.3.271

Johnsen, B. H., Thayer, J. F., Laberg, J. C., Wormnes, B., Raadal, M., Skaret, E., Kvale, G., \& Berg, E. (2003). Attentional and physiological characteristics of patients with dental anxiety. Journal of Anxiety Disorders, 17, 75-87. doi:10.1016/S0887-6185(02)00178-0

Krypotos, A., Jahfari, S., van Ast, V. A., Kindt, M., \& Forstmann, B. U. (2011). Individual differences in heart rate variability predict the degree of slowing during response inhibition and initiation in the presence of emotional stimuli. Frontiers in Psychology, 2, 1-8. doi:10.3389/fpsyg.2011.00278

Lang, P. J., Bradley, M. M., \& Cuthbert, B. N. (2008). International affective picture system (IAPS): Affective ratings of pictures and instruction manual. Technical Report A-8. Gainesville, FL: University of Florida.

Melzig, C., Weike, A., Hamm, A., \& Thayer, J. (2009). Individual differences in fear-potentiated startle as a function of resting heart rate variability: Implications for panic disorder. International Journal of Psychophysiology, 71, 109-117. doi:10.1016/j.ijpsycho.2008.07.013

Porges, S. W. (2007). The polyvagal perspective. Biological Psychology, 74, 116-143. doi:10.1016/j.biopsycho.2006.06.009

Porges, S. W., \& Byrne, E. A. (1992). Research methods for measurement of heart rate and respiration. Biological Psychology, 34, 93-
130. doi:10.1016/0301-0511(92)90012-J

Ruiz-Padial, E., Sollers, J. J., Vila, J., \& Thayer, J. F. (2003). The rhythm of the heart in the blink of an eye: Emotion-modulated startle magnitude covaries with heart rate variability. Psychophysiology, 40, 306-313. doi:10.1111/1469-8986.00032

Russell, J. A., Weiss, A., \& Mendelsohn, G. A. (1989). Affect Grid: A single-item scale of pleasure and arousal. Journal of Personality and Social Psychology, 57, 493-502. doi:10.1037/0022-3514.57.3.493

Thayer, J. F., \& Brosschot, J. F. (2005). Psychosomatics and psychopathology: Looking up and down from the brain. Psychoneuroendocrinology, 30, 1050-1058. doi:10.1016/j.psyneuen.2005.04.014

Thayer, J. F., Friedman, B. H., Borkovec, T. D., Johnsen, B. H., \& Molina, S. (2000). Phasic heart period reactions to cued threat and nonthreat stimuli in generalized anxiety disorder. Psychophysiology, 37, 361-368. doi:10.1111/1469-8986.3730361

Thayer, J. F., \& Lane, R. D. (2000). A model of neurovisceral integration in emotion regulation and dysregulation. Journal of Affective Disorders, 61, 201-216. doi:10.1016/S0165-0327(00)00338-4

Thayer, J. F., \& Lane, R. D. (2009). Claude Bernard and the heart-brain connection: Further elaboration of a model of neurovisceral integration. Neuroscience Biobehavioral Review, 33, 81-88. doi:10.1016/j.neubiorev.2008.08.004

Waugh, C. E., Thompson, R. J., \& Gotlib, I. H. (2011). Flexible emotional responsiveness in trait resilience. Emotion, 11, 1059-1067. doi:10.1037/a0021786

Westphal, M., Seivert, N. H., \& Bonanno, G. A. (2010). Expressive flexibility. Emotion, 10, 92-100. doi:10.1037/a0018420 